\title{
Digital Earth for Smart Municipalities
}

\author{
Eugene Eremchenko ${ }^{1}$ and Ilya Rylskiy ${ }^{1}$ \\ ${ }^{1}$ Lomonosov Moscow State University, GSP-1, Moscow, 119991, Russia
}

\begin{abstract}
The paper discusses the prospects of integrating the concepts of Digital Earth and Smart Cities to achieve synergy and improve global governance by harmonizing decision supports at different scales. Achieving this goal requires overcoming the scale-dependent differentiation of information systems and thus integration of Smart Cities with their geospatial context. A possible approach can be based on the unique property of spatial and temporal localizations their invariance for all subjects. Spatial and temporal localizations are fundamentally different from classical "thematic" ontologies, inevitably contradictory and relative. Implementing the concept of "ontological pluralism" will allow the seamless integration of heterogeneous information into a single spatial and temporal volume. The urgency of practical implementation of such systems is demonstrated briefly against the background of the COVID-19 pandemic and the need to find new approaches to the processing and analyzing environmental factors.
\end{abstract}

\section{Keywords}

Smart City, Digital Earth, governance, decision making, ontological pluralism

\section{Introduction}

Smart Cities and Digital Earth concepts have a lot in common. Both emerged from the same source - the digital transformation that began at the end of the 20th century. Digital Earth was proposed in 1992-1998 [1, 2]. It became a reality in 2005 with the launch of Google Earth online service which, in turn, initiated the active development of the Smart City concept [3]. Both concepts address the problem of the formation of virtual decision-support environments for governance and their implementation in real life.

But their extents are different. Smart City is focused on the metropolises. Instead, Digital Earth pretends to be a single global omniscale management system, combining in a single data set all levels at once - from global to local ones [4]. This contradiction prevents the integrity of governance.

Therefore, developing an integrated approach to combine both concepts is required. Its importance was clearly demonstrated during the COVID-19 pandemic. The inability of existing systems to assess the nature of the threat and its contributing factors in a timely manner and to develop necessary countermeasures against it resulted in the development of a global crisis which, in turn, revealed the inadequacy of the existing crisis response systems.

The main prerequisite for such a crisis development was the absence of a unified holistic and global information structure which would allow to identify in advance the factors and threats of principally new classes. Such a hypothetical hierarchical structure should represent the whole variety of data about the world in their interconnection. It is called "top level ontology". Approaches to the development of the top level ontology are intensively researched [5], but it is very far from being created because it "tantamount to the question of the most basic categories" [6]. Moreover, it is necessary to integrate all possible scales in order to avoid the fragmentation of information images and the consequent generation of inconsistent decisions at different levels of the hierarchically organized control system.

In spite of the worldview unwillingness of modern science to create a top level ontology, its practical necessity is obvious to manage and detect promptly any threats and risks, especially if their nature and

GraphiCon 2021: 31st International Conference on Computer Graphics and Vision, September 27-30, 2021, Nizhny Novgorod, Russia EMAIL: eugene.eremchenko@gmail.com (E. Eremchenko), rilskiy@mail.ru (I. Rylskiy) ORCID: 0000-0002-4416-7617 (E. Eremchenko), 0000-0002-7675-4621 (I. Rylskiy)

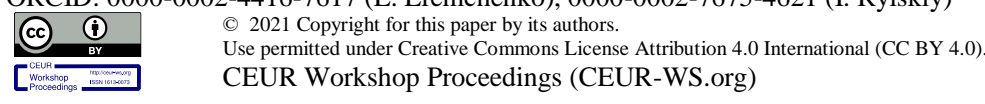


place in the existing ontological schemes are not yet clear. Since the only sufficient platform for global management systems is the Digital Earth, it is necessary to propose principles for organizing information within it.

Data sets for all different scales should be consistent according to the Digital Earth paradigm. Since cities and metropolises are the main habitats of modern mankind and the main source and recipient of technogenic threats, it is necessary to identify the conceptual limitations, both explicit and implicit, that hinder the integration of Digital Earth and Smart Cities and hide behind the ambiguous terms "digital" and "smart", in order to solve the task at hand.

\section{Methodology}

Formally the problem of creating a top level ontology belongs to computer science. Still, in essence, it is a fundamental interdisciplinary worldview problem since it requires the integration of data relating to all research fields without exception. The solution to such a problem requires the consolidation and structuring of all knowledge accumulated by humanity. Indeed, this task cannot be solved, at least in the foreseeable future. At the same time, the critical urgency of ensuring at least elementary expedient management requires creating such a system in a concise time frame. It requires the consolidation of all information in the proper framework, like Digital Earth. Because of the interdisciplinary and fundamental nature of the problem, these principles must satisfy the criteria for a good scientific theory [7].

The task is supposed to use a set of standard research methods, among which integral method, which is an alternative to the differential method widely used in scientific research should be mentioned separately. The integral research method is rarely used in geoinformatics, but $t$ t has been used with astonishing efficiency by the famous endocrinologist Hans Selye (1907-1982). In contrast to the usual approach of identifying disease-specific symptom complexes, he formulated the opposite task and began to study the unspecific responses of the living species to any possible demand of any nature. This allowed him to develop the theory of stress, or general adaptation syndrome, as a universal, non-specific response of living organisms to all kinds of challenges [8]. The approach used by $\mathrm{H}$. Selye has a general scientific significance [9] and can therefore be applied to the task at hand. Accordingly, the study of the global phenomenon through the identification of common properties of its manifestations will be an important goal of the study.

\section{Research and Discussion}

The core concept of a Smart City is an expression of the idea that it is possible and necessary to build a human-compatible technological environment with the help of new technologies. It is focused primarily on large metropolitan areas. To some extent, this peculiarity of perception can be traced by the use of "smart" terms related to entities on different scale levels. Obviously, entities associated with the rural environment are less frequently characterized as "smart" compared to entities associated with the urban environment and new technologies (Figure 1). We can see that the frequency of use of such terms for the less urbanized areas (towns and villages) is relatively low, despite the quantitative predominance of towns and villages over cities and metropolises.

This approach is relatively justified by the fact that urbanization in the modern world is increasing, and the share of the population living in cities is rising constantly. Cities are also centers of technological development and implementation, leading to the fast growth of the risks associated with them. Megalopolises are migration portals, technological and scientific centers and transportation hubs, and so are particularly vulnerable to technological, biological and social hazards. 


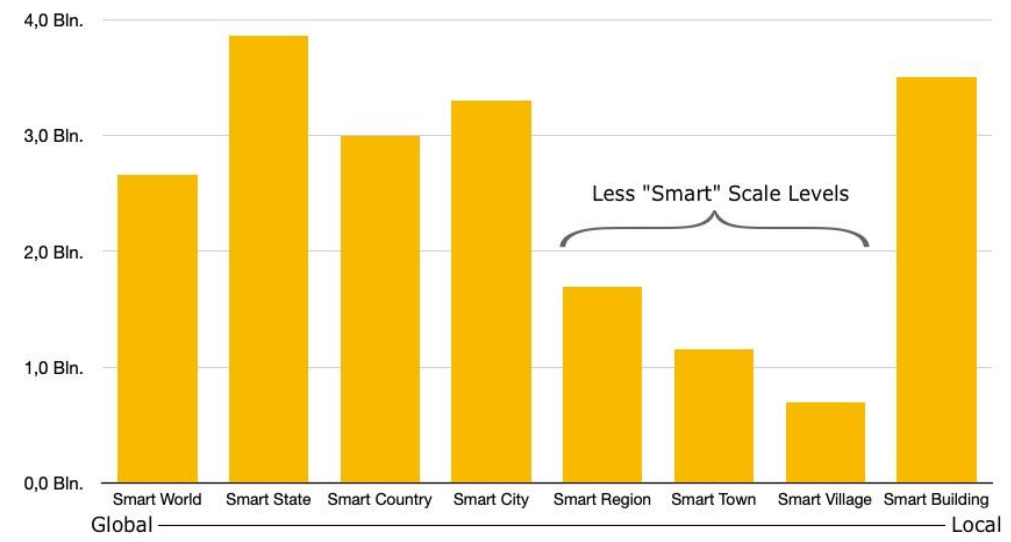

Figure 1: Cumulative number of uses of "Smart" terms of different geospatial entities (according to Google)

It is natural to believe that metropolises should be "smart" and "sustainable" [10]. However, the content of the concepts of "smart" and "sustainability" remains a matter of debate, as the human environment is obviously not limited to the boundaries of large metropolises. Natural factors beyond the control of technological means have not gone out of our world with the advance of urbanization, nor have the threats associated with them diminished. As the COVID-19 pandemic has shown, the threat is even obviously growing $[11,12]$. Areas and waters outside urban islands continue to have an increasing impact on social-environmental processes [13], in particular through the so-called environmental, or ecological factor, and already the applicability of the term "smart" for confined islands is debatable to say the least. Digital Earth makes it possible to overcome this limitation by creating global scale-independent datasets. Digital Earth-based municipal information systems have the following important features that distinguish them from all other geospatial products:

1. The city image in Digital Earth is a global "map for the city" instead of a "map of the city" like an ordinary map and GIS. It is not isolated from the global context by map boundaries and represents the entire planet as a "neighbourhood" of the city. Thus the fragmentation of the environment image by the scale and by extent is excluded.

2. The city image in Digital Earth is no longer confined to a discrete set of thematic layers. Instead, a rich and unmediated geospatial context in the form of images eliminates aberrations, allows the implementation of a situational awareness [14] and the achieving of the qualitative leap in decision making. The elimination of discretization into thematic layers makes it possible to identify entities outside the existing schemes and thus regains the information richness inherent in the real world.

It can be said that the main distinguishing feature of Digital Earth as a sign-less representation [15] is the absence of a rigid and inevitably limited classification schemes that are an inherent property of the maps. Instead, users could percept the environment directly through the senses.

The practical implementation of both of these features requires consideration of new approaches to data representation in information systems. The user should be able to adequately perceive and analyze information that cannot be attributed to any specific layer. This is especially significant when integrating Smart City into Digital Earth and responses the new challenges, threats and risks. In general, they may be new, atypical, and their causes and factors may be implicit. A typical example of such kind of situations is biological threats, such as the pandemic caused by the COVID-19 virus. Without basic definitions (health, disease, life, infection, etc.) and the lack of understanding of the factors contributing to a pandemic, providing information to decision-support systems is challenging. At the same time, the urgent need to address such challenges is evident.

For approaching this issue, it is necessary to consider one of the unusual features of the subject's perception of external reality. The whole set of properties describing reality can be divided into three groups: 1 . What? 2. Where? 3. When? The answer to the first question is always subjective. In situations of intersubjective interaction, it is possible to successfully implement contradictional concepts about the phenomenon in question. In a situation of inter-subjective conflict, the use of different, contradictory ontological systems is one of the most important tools for achieving goals. 
The answers to the second and third questions, by contrast, are invariant. Even subjects who see the phenomenon differently will have the identical representation of its spatial and temporal localization.

The invariance property of space and time is paradoxical, since both categories, on the one hand, are subjective [16], and the spatio-temporal frameworks for external perceptions are generated by individual subjects independently. On the other hand, it is equally clear that these both categories are generated by all subjects in the very same manner, synchronously and identically. This strange peculiarity of the invariance of individual space and time has no currently accepted explanation and needs to be studied. However, it can already be used to create systems that can identify fundamentally new phenomena and provide their representation in decision support systems.

Such kinds of information systems should be comprehensive replicas of reality. At the same time they must have an open ontological structure, i.e. they would allow to include and display any information in the system instantly, as it recognized and before its strict classification.

The basis for creating such systems should be the requirement of representation of the environment through the senses, bypassing the mediation by signs. Along with the environment image integrity requirement, it is a demand of the situational awareness principle [17]. This can be achieved assuming the spatial and temporal localization ("where?" and "when?") of any information as the only ontologically significant factors inevitably characterizing any information and necessary for its embedding into the spatio-temporal geospatial framework and subsequent analysis. Any thematic ontology will be secondary and, by necessity, relative one (Figure 2). This principle can be described as "ontological pluralism" (in an information sense of ontology), because it presupposes the coexistence of several incompatible ontological models [18].

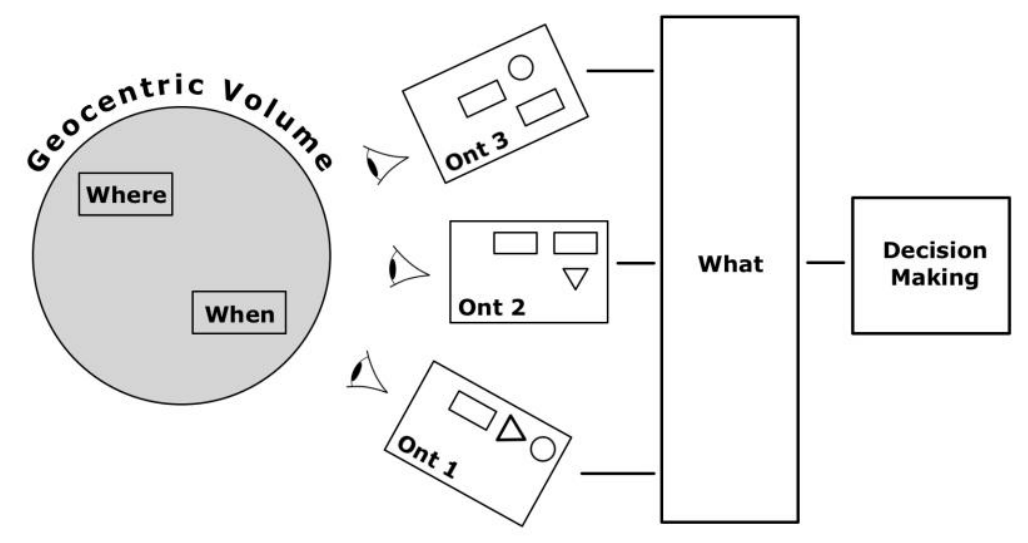

Figure 2: A simplified representation of the principle of information organization in the Digital Earth using the principle of ontological pluralism

Naturally, this approach is primarily applicable to the identification and analysis of fundamentally new objects, phenomena and processes in natural and social systems that are not described and even predicted by existing models. This opens up the possibility of studying such objects, phenomena and processes by their spatial and temporal dynamics, which makes it possible to identify their material carriers and the factors influencing them. Such an approach may be particularly fruitful in the study of unique phenomena existing in geocentric space, such as the nature of living things and processes in living systems, the environmental factor in all its manifestations, social phenomena, etc. The most effective source of data on such processes is modern urbanized areas, united by the term Smart Cities. A significant difference between the proposed approach and the classical view of Smart Cities as a highly automated spaces is that the answer to the question "what?" becomes a consequence of the answers to the questions "where?" and "when?".

The proposed approach based on Digital Earth restores the integrity of the image of geocentric space in information systems, eliminating the dissociation of "smart" cities and their "non-smart" environments. It makes possible to identify relationships that are not detectable with classical scaledependent tools - primarily, correlations between processes occurring at different scales. Such a system 
is equally compatible with different ontological schemes and allows the principle of "ontological pluralism" to be put into practice. A possible structure of a similar system interface implemented in the Google Earth environment is shown in Figure 3.
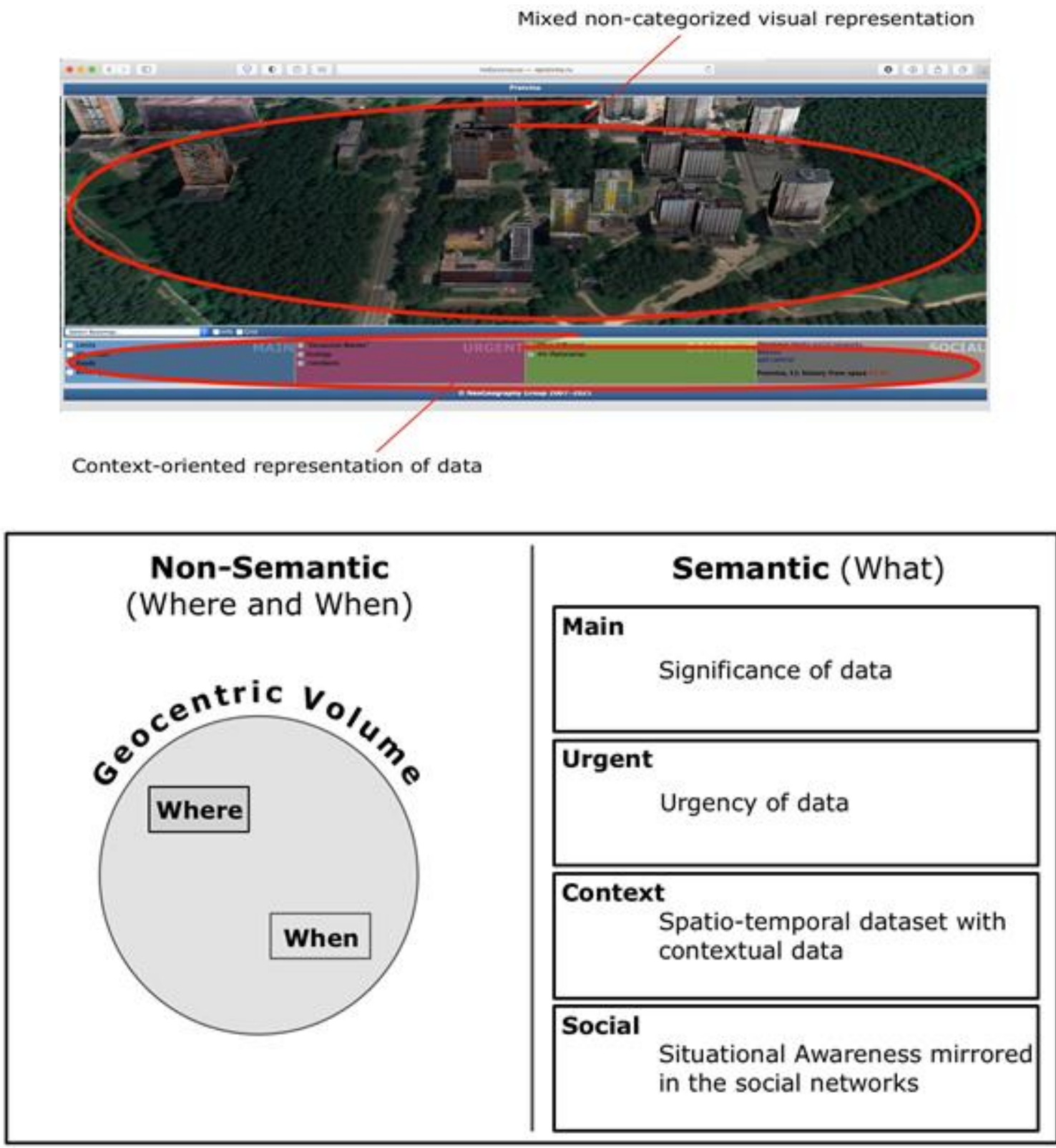

Figure 3: Simplified scheme (below) and possible implementation (above) of the interface of the Digital Earth system with the flexible ontology-independent representation of the data site based on context approach

\section{Conclusions}

The integration of Digital Earth and Smart Cities concepts creates a set of unique opportunities to simultaneously enhance governance by improving decision-making and to use global datasets as a means to study biological, social, and anthropogenic phenomena in their interconnectedness [19]. In this case, the solution of both tasks requires the organization of the representation of the environment, which allows to combine the different scales of data and to realize the hidden potential in them. The basis for such a representation can be the concept of ontological pluralism, implemented through a holistic, rich and unmediated representation of the environment and the inclusion of data sets structured mainly by their spatial and temporal localization. Such an approach is based, on the one hand, on the 
invariance of spatial and temporal localizations and, on the other hand, on the ontological relativity. Ontological pluralism provides unique capabilities for fast response to new challenges, risk ant threats like COVID-19 pandemy.

\section{Acknowledgements}

Research was supported by RSF grant \#20-47-01001.

\section{References}

[1] A. Gore, Earth in the Balance: Ecology and the Human Spirit. New York: Houghton Mifflin Harcourt. 1992. pp. 357.

[2] A. Gore, The Digital Earth: Understanding our planet in the 21st Century. Al Gorespeech at California Science Center, Los Angeles, California, on January 31, 1998.

[3] History of smart cities: Timeline, 2020. URL: https://www.verdict.co.uk/smart-cities-timeline/.

[4] H. Guo, M.F. Goodchild, A. Annoni (eds), Manual of Digital Earth, Springer, Singapore, 2020. doi: 10.1007/978-981-32-9915-3.

[5] A survey of Top-Level Ontologies, 2020. URL: https://www.cdbb.cam.ac.uk/files/a_survey_of_top-level_ontologies_lowres.pdf.

[6] L. Jansen, The Top-Level Ontology, 2008. URL: http://mba.eci.ufmg.br/downloads/pos/TopLevelOnto-Jansen.pdf.

[7] T. Kuhn, Objectivity, value judgment, and theory choice. In: The Essential Tension: Selected Studies in Scientific Tradition and Change. University of Chicago Press, Chicago, 1977.

[8] H. Selye, The Stress of Life, McGraw-Hill Book Company, New York, 1956.

[9] H. Selye, From Dream to Discovery. On Being a Scientist, McGraw-Hill Publishing Company Limited, London, 1964.

[10] Goal 11: Make cities inclusive, safe, resilient and sustainable, 2021. URL: https://www.un.org/sustainabledevelopment/cities/.

[11] COVID-19 dashboard by the Center for System Science and Engineering (CSSE) at John Hopkins University (JHY), 2021. URL: https://gisanddata.maps.arcgis.com/apps/dashboards/bda7594740fd40299423467b48e9ecf6.

[12] W. Leal Filho, A.M. Azul, T. Wall, et al., COVID-19: the impact of a global crisis on sustainable development research, Sustain Sci 16 (2021) 85-99. doi: 10.1007/s11625-020-00866-y.

[13] H. Eakin, A.L. Luers, Assessing the Vulnerability of Social-Environmental Systems, Annual Review of Environment and Resources, 31(1) (2008) 365-394. doi: 10.1146/annurev.energy.30.050504.144352.

[14] E. Eremchenko, V. Tikunov, Defnition of Digital Earth and main conundrum of cartography, IOP Conference Series: Earth and Environmental Science, 509(1) (2020) 012015.

[15] V. Tikunov, E. Eremchenko, Digital Earth and Cartography (Russian), Geodesy and Cartography, 11 (2015) 6-15.

[16] I. Kant, Critique of Pure Reason, 1781. Translated by: N.K. Smith, URL: http://staffweb.hkbu.edu.hk/ppp/cpr/toc.html.

[17] M. Endsley, Toward a theory of situation awareness in dynamic systems, Human Factors, 37 (1995) 36.

[18] M. Durante, The Value of Information as Ontological Pluralism. Know Techn Pol 23 (2010) 149161. doi: 10.1007/s12130-010-9105-9.

[19] S.A. Umpleby, T. Medvedeva, V. Lepskiy, Recent Developments in Cybernetics, from Cognition to Social Systems, Cybernetics and Systems, 50(4) (2019) 367-382. doi: $10.1080 / 01969722.2019 .1574326$. 